\title{
Looking for lobes in all the right places: Signatures of the interaction of microquasars with the ISM
}

\author{
Heinz, S.* Aloy, M.A., Fender, R., \& Russell, D. \\ University of Wisconsin, Madison; E-mail: heinzs@astro.wisc.edu
}

\begin{abstract}
Microquasars are in many ways similar to their massive counterparts, the radio loud population of AGNs. One aspect that is just beginning to receive the due amount of attention is their interaction with the interstellar medium. This interaction is similar on a qualitative level, but different quantitatively: The interstellar medium provides a much weaker barrier to microquasar jets than the intergalactic medium does to AGN jets. As is the case in AGN jets, the interaction of microquasar jets with the ISM should manifest itself through three observable channels, which we explore sequentially in this article: (a) the inflation of radio lobes, (b) the production of dense shells of swept up ISM around those lobes, and (c) the direct interaction of radio plasma with the ISM in the form of a working surface or hot spot. We present simple scaling relations that can be used as guides to design search campaigns and to analyze existing observations of lobes and shells around microquasars.
\end{abstract}

VI Microquasar Workshop: Microquasars and Beyond September 18-22 2006

Società del Casino, Como, Italy

\footnotetext{
${ }^{*}$ Speaker.
} 


\section{Introduction}

\subsection{The historical picture: Radio galaxies}

Extragalactic radio sources have been studied for sixty years. We understand what causes the extended synchrotron emission in radio galaxies: jet exhaust, that has traveled sometimes hundreds of kpc from the active galactic nucleus (AGN), interacted with the intergalactic medium (IGM), and inflated a cocoon or radio lobe of relativistic plasma. The interaction of these AGN jets with the IGM often occurs directly at the end of the jet (this is true for the more powerful FanaroffRiley type II sources Fanaroff \& Riley, 1974) in the form of a terminal shock, often called working surface or simply hot spot.

The past decade has seen a new trend complementing the radio picture in the numerous spectacular Chandra observations of X-ray cavities in galaxy clusters (e.g. Bîrzan et al., 2004). The cavities are spatially coincident with the lobes of central cluster radio galaxies and are framed by bright shells of dense, thermally emitting cluster gas that was swept up by the expanding radio lobe.

We routinely use these cocoons and cavities to measure jet power, constrain jet composition, and to study jet dynamics (e.g., Heinz et al., 2002; Choi et al., 2004). At the same time, it has become clear that AGN jets are the primary feedback mechanism for growing black holes: While they may not carry as much total kinetic energy as the integrated bolometric energy output of a growing black hole (the jet production efficiency of accreting black holes is still a poorly known quantity), the IGM is entirely optically thick to this energy output - as opposed to the radiation emitted by the black hole in its quasar phase (most of which escapes the galaxy and the galaxy cluster within which it was emitted). In other words: All of the kinetic energy released by the black hole is transferred to the IGM. Thus, black holes and the jets they produce have taken on a new, important role in the larger picture of structure formation.

\subsection{Microquasars}

The discovery of relativistic jets from GRS 1915+105 (Mirabel \& Rodríguez, 1994) and GRO J1655-40 (Hjellming \& Rupen, 1995) had a number of drastic implications for our understanding of black hole accretion and jet production. Most importantly, intense work over the past decade has lead to realization that virtually every Galactic black hole X-ray binary (XRB) produces powerful jets for a fraction of its life has, i.e., every black hole XRB is a microquasar (Fender \& Kuulkers, 2001).

Large scale jets and compact, flat spectrum radio cores are frequently observed in a number of $\mathrm{XRBs}$, though at significantly lower fluxes than their counterparts produced by supermassive black holes in AGNs (for a quantitative reason for why that is, see, e.g., Heinz \& Sunyaev 2003). These are the morphological analogs to kpc scale jets and AU-scale radio cores in objects like M87 and Cygnus A. As in the case of AGN jets, these jets transport relativistic particles and magnetic fields in a narrow, collimated channel.

If these jets are so common, the natural question to ask is: What happens to the jet plasma when it reaches the interstellar medium (ISM)? It is clear that the jet must interact with the ISM in some way. Given the obvious similarities between microquasars and radio loud AGNs in their core 
properties that indicate a scale invariance between the two types of jets (Heinz \& Sunyaev, 2003), one might be tempted to assume that a similar scale invariance holds for the large scale structure of jets and their interaction with the ISM.

However, this is not the case. To see this, let us consider the quantities that are relevant for the interaction of the jet with the ISM: The jet power $P$, the jet cross sectional area $A_{\text {jet }}=\pi R^{2}$, and the density $\rho_{\text {env }}$ and pressure $p_{\text {env }}$ of the environment (or, equivalently, its sound speed $c_{\mathrm{s}}=$ $\left.\sqrt{5 p_{\text {env }} / 3 \rho_{\text {env }}}\right)$. These quantities form a dimensionless number

$$
\eta=\frac{P / \pi R^{2}}{\rho_{\mathrm{ISM}} c_{\mathrm{S}}^{3}}
$$

which sets the characteristic scales for the interaction. $\eta$ is simply the ratio of jet thrust to the characteristic ISM dynamical pressure. The jet-environment interaction can only be scale invariant between AGNs and microquasars if this number is the same in both cases. It is easy to see that it is not: The jet power will be proportional to the mass of the central black hole $M_{\mathrm{BH}}$ (typically up to a few percent of the Eddington power), the jet cross section is set by the size scale of the jet production region, which should be of order 10-100 gravitational radii, so $R^{2} \propto M_{\mathrm{BH}}^{2}$.

For $\eta$ to be identical in AGNs and microquasars, one would require $\rho_{\mathrm{env}} c_{\mathrm{s}}^{3} \propto M_{\mathrm{BH}}^{-1}$. While typical ISM densities are always larger than those of the IGM, the sound speed is always lower, especially in regions with large densities (i.e., molecular environments) and $\eta_{\text {microquasar }}$ is always at least 4 orders of magnitude smaller than $\eta_{\mathrm{AGN}}$.

The immediate consequence is that the ISM is under-pressured and under-dense relative to microquasar jets when compared dynamically to AGN jets in the IGM. In other words: the ISM provides a much weaker barrier to microquasars than the IGM does for AGN jets. Thus, microquasar jets and lobes should propagate further and more quickly than AGN jets when measured in central engine units (i.e., dynamical times and $r_{\mathrm{g}}$ ). One might further speculate that their lobes should be dimmer as a result, which we will show below.

\subsection{Morphology}

While we argued above that the interaction between microquasars and the ISM should be quantitatively different, we can still expect similar physics to hold. The jets move relativistically and the ISM is cold and dense, so there must be a transition from relativistic to sub-relativistic flow somewhere along the jet, most likely in the form of a strong shock (since the jets move supersonically). This shock would be the equivalent of the working surface or hot spot. From this shock, relativistic plasma must spread laterally away from the jet, as is the case in AGN jets, and fill some kind of plasma bubble - a radio lobe. The ISM gas that was previously in the place of the radio lobe must be pushed aside.

This very simple picture is clearly guided by what we know about AGN jets, but it predicts the presence of three distinct physical structures: Radio lobes (though they might not be radio bright, as we will see), hot spots, and ISM shells. The question then is: Are any of these signatures observable? This article shall explore them in sequence. 


\section{Lobes}

In radio galaxies, the extended structure comprised by hot spots and lobes is often the brightest and most luminous part. Clearly, microquasars were not discovered as static, diffuse radio sources, so their radio brightness and luminosity distribution must be different. There are, of course, a few exceptions to this rule, most notably SS 433 , which has very bright radio lobes. We will see why this might make sense in a few paragraphs.

The same arguments used in the previous section about scaling have been put forward to derive a self similar solution of luminosity driven bubble expansion (Castor et al., 1975; Heinz et al., 1998). This solution is the functional equivalent of the Sedov-Taylor solution for a blast wave in the case of continuous energy supply and is valid generally for spherically symmetric external density profiles that follow a power-law shape. In the case of microquasars, we will assume that the ISM is homogeneous, simplifying the solution even further. The solution makes the assumption of a spherically expanding bubble, which is not exactly true as radio lobes should be expected to be elliptical, however, the scalings of the solution are exact and as long as the aspect ratios of the lobes are of order unity, the solution is accurate enough for quantitative estimates as well. It was first employed for microquasars in (Heinz \& Sunyaev, 2002).

For a kinetic power of $P$ and an external density of $\rho_{\mathrm{ISM}}$, the dynamical solution for the radio lobe radius $R_{\mathrm{L}}$ is (e.g. Heinz et al., 1998)

$$
R_{\mathrm{L}}=0.65\left(\frac{P t^{3}}{\rho_{\mathrm{ISM}}}\right)^{\frac{1}{5}}
$$

The swept up shell in the absence of any cooling has a thickness of $10 \%$ of the shell radius, which is easily understood from the strong shock compression of the swept up gas to $25 \%$ of its original volume. The shell radius is denoted below as $R_{\mathrm{S}}$.

It is important to note here that $P$ is the time averaged power: The expansion time of lobe is much longer than the variability time scale of jets from microquasars for sufficiently large, mature lobes. Let us parameterize the life time of the XRB as $t=t_{5} 10^{5} \mathrm{yrs}$ in units of $10^{5} \mathrm{yrs}$, which is short compared to the life time of most binaries but long compared to the variability (or transient) time of the binary. It might give us a good idea of what to expect for young sources like Cygnus $\mathrm{X}-1$.

It is further useful to parameterize the time average power $P=P_{36} 10^{36} \mathrm{ergs} \mathrm{s}^{-1}$ in units of $10^{36} \mathrm{ergs} \mathrm{s}^{-1}$. Finally, we will take the canonical ISM density of $1 \mathrm{~cm}^{-3}$ and write $n=n_{1} \mathrm{~cm}^{-3}$. We can then write the radius of the lobe as

$$
R_{\text {lobe }}=6 \mathrm{pc} P_{36}^{0.2} n_{1}^{-0.2} t_{5}^{0.6}
$$

which is very large compared to binary scales or even the jet core. If we take a typical distance to the source to be $D=D_{1} 1 \mathrm{kpc}$, the angular size of such a lobe would be

$$
\mu_{\text {lobe }}=3.3^{\circ} P_{36}^{0.2} n_{1}^{-0.2} t_{5}^{0.6} D_{1}^{-1}
$$

The radio lobe fills the inner $75 \%$ of the volume, out to a radius of $R_{1}=0.9 R_{\mathrm{s}}$. The pressure inside the shell and the lobe is equal to the ram pressure of the ISM (this is simply momentum 
conservation):

$$
p=\rho_{\mathrm{ISM}} \dot{R}^{2} \approx 2.6 \times 10^{-11} \mathrm{ergscm}^{-3} P_{36}^{0.4} n_{1}^{0.6} t_{5}^{-0.8}
$$

With these expressions in place, we can estimate the order of magnitude of the synchrotron emission from the lobe, for now neglecting effects of radiative cooling. Using the simplified expression for the synchrotron emissivity from eq. (1) in Heinz (2006) we can write

$$
j_{v}=\frac{3.1 \times 10^{-17} \mathrm{ergs}}{\mathrm{cm}^{3} \mathrm{~Hz} \mathrm{~s}} v_{5 \mathrm{GHz}}^{-1 / 2} f_{p, B}
$$

where $f_{p, B}$ contains corrections for deviations from equipartition for protons and magnetic fields relative to the electron pressure. This gives a synchrotron luminosity of

$$
L_{v} \approx 2.4 \times 10^{23} \mathrm{ergs} \mathrm{Hz}^{-1} \mathrm{~s}^{-1} P_{36}^{1.3} t_{5}^{0.4} n_{1}^{0.45} f_{p, B} v_{5}^{-0.5}
$$

Since radiative losses are not taken into account here, this is an upper limit. At low frequencies, this expression should be rather robust, however. The quantity most relevant for detectability is the radio surface brightness. We approximate it as

$$
S_{V} \approx 50 \frac{\mu \mathrm{Jy}}{\operatorname{arcsec}^{2}} P_{36}^{0.9} t_{5}^{-0.8} n_{1}^{0.85} f_{p, B} v_{5}^{-0.5}
$$

The dependence on time, power, and ISM density is most enlightening: The more powerful the source, the younger the source, and the denser the environment, the larger its surface brightness will be. On the other hand, the total luminosity depends on a positive power of the source age. Thus, depending on what kind of instrument is involved, one might be more sensitive to young or old sources.

This also clarifies why SS433 should be easily visible: It is by far the most powerful microquasar we know of today, with a kinetic power of order $P \gtrsim 10^{39} \mathrm{ergs} \mathrm{s}^{-1}$. In fact, the radio luminosity of the source is rather low for the canonically assumed power and age. Given reasonable assumptions about the environment, the source should be even much brighter. There are only a few possible conclusions: (a) the time averaged kinetic power is much lower than the estimate based on the currently observed optically emitting jets, (b) the bubble is running into an evacuated cavity and is much younger than assumed, and/or (c) the source suffers from significant cooling, thus indicating that it must be out of equipartition.

Radiative cooling, under the assumption of uniform magnetic pressure and an isotropic pitch angle distribution, will produce a spectral break of $\Delta \alpha_{v}=1 / 2$. The break frequency $v_{\mathrm{c}}$ is roughly located at

$$
v_{\mathrm{c}} \approx 1.5 \times 10^{13} \mathrm{~Hz} P_{36}^{-0.6} n_{1}^{-0.9} t_{5}^{-0.8} f_{B}^{-1.5}
$$

We can see that cooling is only going to be important for old, powerful sources and those that are in dense environments. Below this break, the luminosity and surface brightness are given by eqs. (2.6) and (2.7). Above the break, these expressions have to be corrected by the factor $\left(v / v_{c}\right)^{-1 / 2}$.

It is clear, then, that radio lobes are most easily detected around sources that are high power, young, and in high density environments. Sources with detected lobes include SS433 (Dubner et al., 1998), Cir X-1 (Fender et al., 1998), 1E1740.7-2942 (Mirabel et al., 1992), and GRS 1758258 (Hardcastle, 2005). It is not unreasonable to speculate that these sources fulfill some or all of 
these requirements. It is clear, however, that other sources do not show direct radiative evidence for the presence of lobes, possibly due to the low density of their environment. The question then becomes: Are there other ways of detecting them?

\section{Shells}

Inflating a radio lobe necessarily implies that the material that was previously in the place of the radio plasma must be pushed aside. As is well known from X-ray cavity studies, this material is initially swept up in a shell (e.g. Reynolds et al., 2001, 2002). In the model laid out above, the thickness of this shell is about $10 \%$ of the radius of the bubble. If the radio emission from the lobe itself is not detectable, the question becomes: Is the shell detectable instead?

The past year has seen the answer to this question: The first shell of this kind was detected around Cygnus X-1 (Gallo et al., 2005; Russell et al., 2007). As is well known from AGN lobes, one can use the detection of one of these shells to determine the source properties. Another possible detection around GRO J1655-40 was reported in these proceedings by Russell. We refer the reader to those publications for a detailed description of the observations.

Like lobes, shells should follow roughly the same self similar solution introduced in the previous chapter. Thus, measuring shell size and expansion velocity allows us to calculate the age of the source and the ratio of $P / \rho_{\text {ISM }}$ (e.g., in the case of Cygnus X-1, the X-ray and radio measurements indicate an expansion velocity of $100 \mathrm{~km} \mathrm{~s}^{-1}<v<300 \mathrm{~km} \mathrm{~s}^{-1}$ Russell et al., 2007). We will express all quantities in the fiducial units defined above.

Below, we will ignore the real possibility that the shell is radiative and will only consider free-free emission from the shell (ignoring line emission as well). This is appropriate for radio and $\mathrm{X}$-ray emission, but will badly underestimate the optical emission around classically strong lines like $\mathrm{H}_{\alpha}$, [OIII], and [SII].

From eq. (2.4), we know the shell pressure. For a non-radiative shock, the density behind the shock is simply $4 \rho_{\text {ISM }}$, so the temperature is

$$
T_{\text {shock }} \approx 4.6 \times 10^{4} \mathrm{~K} P_{36}^{0.4} n_{1}^{-0.4} t_{5}^{-0.8}
$$

The total free-free emission from the shell is then

$$
L_{\mathrm{ff}}=10^{36} \mathrm{ergs} \mathrm{s}^{-1} P_{36}^{0.8} n_{1}^{1.2} t_{5}^{1.4}
$$

and the spectral flux is

$$
L_{v, \mathrm{ff}}=3 \times 10^{18} \mathrm{ergs} \mathrm{s}^{-1} \mathrm{~Hz}^{-1} P_{36}^{0.4} n_{1}^{1.2} t_{5}^{2.3} e^{-\frac{v_{\mathrm{keV}}}{1.6} n_{1}^{0.4} t_{5}^{0.8} P_{36}^{-0.2}}
$$

The maximum free-free surface brightness through the shell will be given at sight lines tangential to the radio lobe, where, for a shell thickness of $10 \%$, the path through the shell is $\Delta y=0.82 R_{\mathrm{s}}$ :

$$
S_{\max }=2.2 \times 10^{-15} \mathrm{ergs} \mathrm{s}^{-1} \operatorname{arcsec}^{-2} P_{36}^{0.4} n_{1}^{1.6} t_{5}^{0.2}
$$

and the spectral surface brightness reaches

$$
S_{v, \text { max }}=1 \mu \mathrm{Jy} \operatorname{arcsec}^{-2} t_{5} n_{1} e^{-\frac{v_{\mathrm{keV}}}{1.6} n_{1}^{0.4} t_{5}^{0.8} P_{36}^{-0.2}}
$$


at the inner edge of the shell.

A full treatment of line emission and non-thermal emission from the shell is beyond the scope of this proceedings article, however, we can treat the extreme case of a fully radiative shock without the need to know the details in the same way that cooling flows are treated in X-ray spectral modeling: We can very simply estimate the bolometric luminosity from mass conservation:

$$
L_{\mathrm{Bol}}=3 \times 10^{35} \mathrm{ergs} \mathrm{s}^{-1} P_{36}
$$

(the partition of energy between shell and lobe is governed by the adiabatic indices of the two, allocating $30 \%$ of the free energy to radiation).

All of these scalings will be useful in the coming years as more and more shell observations become available. It is clear that, once again, powerful sources located in dense environments will be easier to detect by their shells than ones in low density environments, biasing against sources in low density settings. There is, however, an indirect way of detecting radio lobes that does not select against sources in low density settings, which we will discuss next. In analogy to faint or dark fossil radio lobes around AGNs (called radio ghosts), such structures might be called micro-ghosts

\section{Jet termination and the dynamical evidence for the existence of micro ghosts}

As discussed above, the jet material must slow down from relativistic speed when entering the lobe. This can occur inside the lobe itself (as is the case in FR I type sources Fanaroff \& Riley, 1974) or in a hot spot / working surface. The deceleration is generally due to the interaction with the ambient medium, either through entrainment or a terminal shock. In either case, enough material must be swept up to slow down the jet material. Given limits on the matter content of the jet, we can thus use the observed jet kinematics to put limits on the amount of matter swept up, and thus, on the density of the material the jet is traveling through. If these limits are well below normal ISM densities, we can conclude with confidence that the jet must be traveling through an evacuated region.

A detailed discussion of the dynamics of decelerating relativistic beams/ejections is beyond the scope of this article. We will use two simple lines of argument and refer the reader to a future publication (Heinz \& Aloy, in preparation) for more details.

\subsection{Dynamical friction}

The first argument was already laid out in Heinz et al. (2002): An amount of jet material (subsequently termed "ejection") with mass $M_{\text {jet }}$ traveling with relativistic speed given by its Lorentz factor $\Gamma=\sqrt{1 /\left[1-(v / c)^{2}\right]}$ must sweep up or plow through an amount of gas with mass $M_{\text {slow }}=M_{\text {jet }} / \Gamma$ in order to slow down appreciably (i.e., reduce its Lorentz factor by a factor of, say, $2)$. For a jet cross section of $A_{\text {jet }}=\pi R_{\text {jet }}^{2}$, this translates to a travel distance of $D=M_{\text {slow }} /\left(\pi R^{2} \rho_{\text {env }}\right)$.

If the jet does not slow down within an observed distance $z$ from the binary, we can thus put an upper limit of $\rho_{\mathrm{env}}<M_{\mathrm{jet}} /\left(\Gamma z \pi R^{2}\right)$ on the density.

Jet collimation is an unsolved problem and it is not clear how microquasar jets travel: They could be ballistic, in which case their cross section evolves under free, supersonic expansion, or they could be internally or externally collimated. For a ballistic jet / ejection that has not slowed 
down, we can assume that the jet has a constant opening angle $\alpha$ (as the opening angle only changes due to changes in velocity). The limit on the density can then be rewritten as

$$
\rho_{\mathrm{env}}<\frac{M_{\mathrm{jet}}}{\Gamma z^{3} \pi \alpha^{2}}
$$

Below, we will concentrate on the case of GRS 1915+105 for illustrative purposes only. Other sources are available in the literature for similar analyses, such as GRO J1655-40 (Hjellming \& Rupen, 1995) and XTE J1550-564 (Corbel et al., 2002).

Fender et al. (1999) resolved optically thin collimated ejections in GRS 1915+105 with MERLIN to travel out to an angular distance of 300 mas from the XRB without any sign of slowing down. In the process, the flux from the ejections decreased monotonically, with the south-eastern ejection eventually reaching an $8.4 \mathrm{GHz}$ flux of $300 \mu \mathrm{Jy}$ at a distance of $\mu=30$ mas. Based on the distance estimate of $11 \mathrm{kpc}$ to the source, they derived a Lorentz factor of $\Gamma=5$ and a viewing angle of $\theta=60^{\circ}$ for the ejections. However, the true distance to the source is not well known and has been subject of considerable discussion recently (Kaiser et al., 2004). Thus, we will carry the distance through our analysis as an unknown parameter.

The radio light curve of the individual ejections show that they are still in free expansion when they reach the sensitivity threshold, consistent with a spherical bullet in adiabatic expansion. This indicates that the bullets are still ballistic all the way through the end of the observation. The ejections also maintain a constant velocity throughout the MERLIN observation, indicating the they do not slow down. We can thus assume that the bullets have a constant opening angle $\alpha$.

We will adopt the constraints on jet speed and viewing angle from Fender et al. (1999), specifically

$$
\tan (\theta)=0.2(D / 1 \mathrm{kpc})
$$

and

$$
\beta=0.41 / \cos (\theta)
$$

For an observed transverse distance $l=300 \mathrm{mas} D=4.5 \times 10^{15} \mathrm{~cm}(D / 1 \mathrm{kpc})$, the true, unprojected travel distance of the ejection is then $z=300 \mathrm{mas} D / \sin (\theta)$. Together, $\Gamma$ and $\theta$ also provide the Doppler factor $\delta=[\Gamma(1-\beta \cos (\theta))]^{-1}$ of the approaching ejection.

The mass of the synchrotron emitting blob can be estimated from the amount of synchrotron radiation. Given the lack of knowledge of jet composition, we can parameterize the departure from equipartition as $f_{\mathrm{p}} \equiv p_{\text {particles }} / p_{\text {tot }} \leq 1$ and the fraction of inertia in synchrotron emitting particles vs. dark particles (like protons) as $f_{\mathrm{M}} \leq 1$. Assuming the ejection is roughly spherical (consistent with its adiabatic power-law decline in flux), we can estimate the volume as $V_{\mathrm{b}} \approx 4 \pi / 3 \alpha^{3} z_{\mathrm{b}}^{3}$.

Given the observed flux of $300 \mathrm{mJy}$, this translates to an estimated internal pressure $p_{\text {int }}$ of

$$
p_{\text {int }} \approx \frac{8 \times 10^{-9} \operatorname{ergs~cm}^{-3} D_{1}^{8 / 7}}{f_{\mathrm{p}}^{4 / 7} \alpha^{12 / 7}\left(1+0.04 D_{1}^{2}\right)^{6 / 7}\left(0.832-0.0067 D_{1}^{2}\right)}
$$

the internal mass is then

$$
M_{\mathrm{b}}=\frac{3 p V_{\mathrm{b}}}{f_{\mathrm{M}} c^{2}}
$$




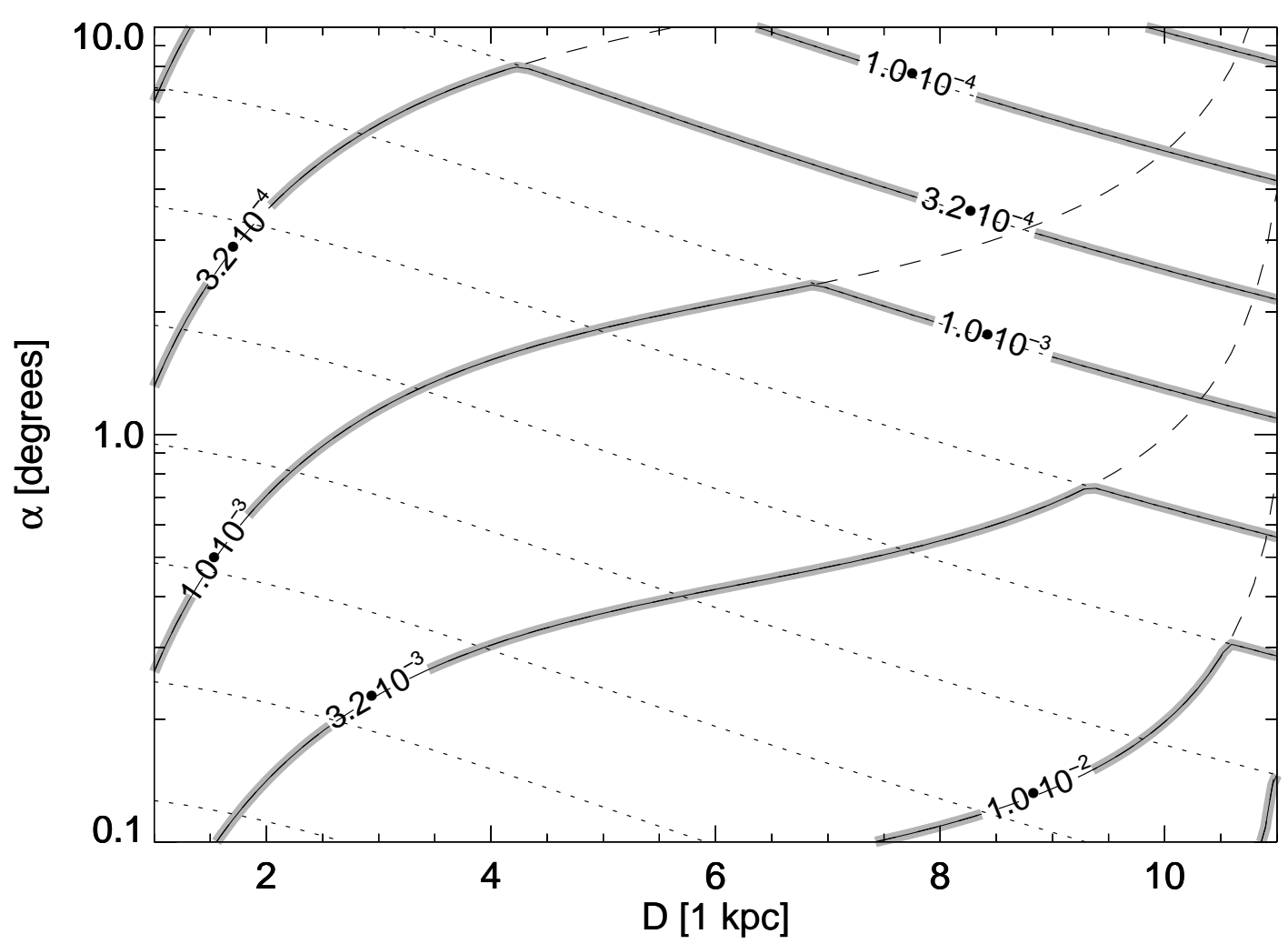

Figure 1: Upper limit on density, in particles per $\mathrm{cm}^{3}$ of the immediate environment of GRS 1915+105 from dynamical constraints on travel distance and ram pressure collimation. Plotted are contours of $n$.

With these constraints, and given the fact that there is no appreciable deceleration in the MERLIN maps, the limit on the external proton density $n$ from eq. (4.1) can be written as

$$
n_{\mathrm{env}}=\frac{\rho}{m_{\mathrm{p}}}<\frac{2 \times 10^{-5} \mathrm{~cm}^{-3} D_{1}^{8 / 7}}{\left(1+0.04 D_{1}^{2}\right)^{6 / 7} f_{M} f_{p}^{4 / 7}\left(0.832-0.0067 D_{1}^{2}\right)^{1 / 2} \alpha^{5 / 7}}
$$

where $m_{\mathrm{p}}$ is the proton mass. This limit is plotted in Fig. 1 .

\subsection{Dynamical collimation}

The ejections in GRS 1915+105 not only maintain their velocity throughout the entire MERLIN observations, they also show no sign of confinement, as their flux continues to drop in powerlaw fashion until they fall below the detection threshold. This implies, as indicated above, that the ejections are in free, ballistic expansion.

As pointed out in Heinz (2000), ram pressure interaction of an ejection with the environment also provides lateral pressure on the ejection itself. If this lateral pressure becomes comparable to the internal pressure of the ejection, it is no longer in free expansion and becomes ram pressure confined. Thus, the observed lack of any collimation/confinement implies that the ram pressure of the environment the ejections are traveling through is smaller than the internal pressure of the 
bullet. Since we have an estimate of the internal pressure of the ejection from the synchrotron flux, we can limit the amount of ram pressure and once again arrive at a limit on the external density.

The ram pressure on an ejection traveling at Lorentz factor $\Gamma$ is simply

$$
p_{\text {ram }} \approx \rho_{\text {env }} \Gamma^{2} \beta c^{2}<p_{\text {int }}
$$

Combined with eq. 2.4 , this provides the following limit on the density:

$$
n_{\mathrm{env}}<\frac{p_{\text {int }}}{\Gamma^{2} \beta^{2} c^{2} \mu m_{\mathrm{p}}}=3 \times 10^{-5} \mathrm{~cm}^{-3} f_{\mathrm{p}}^{4 / 7} \alpha^{-12 / 7}\left(1+0.04 D_{1}^{2}\right)^{-13 / 7}
$$

Contours of the limit on $n_{\mathrm{env}} f_{\mathrm{p}}^{-4 / 7}$ are also shown in Fig. 1 as dotted lines.

Looking at Fig. 1, it is clear that the densities implied by these limits are significantly smaller than the canonical density of the ISM in the disk of our galaxy, with the possible exception of very small opening angles ${ }^{1}$.

Given the ongoing jet activity of the source, the most reasonable explanation for such low densities is that previous episodes of jet production in GRS 1915+105 have inflated a radio lobe, filled with very low density relativistic plasma that provides essentially no barrier to the newly ejected jet plasma. Thus, we can take the dynamical limits on the environmental density as circumstantial evidence of a radio lobe around GRS 1915+105. Other sources, such as GRO J1655-40 and XTE J1550-564, which show similar behavior essentially lead to the same conclusion (though a detailed discussion would go beyond the scope of this article - see Heinz \& Aloy, in preparation).

\section{5. summary}

We presented a short overview of the different manifestations of microquasar-ISM interactions. Three independent lines of evidence exist for this interaction, all of which point to the existence of large scale radio lobes around virtually all black hole X-ray binaries: (a) direct radio detections of radio lobes in a number of systems, (b) thermal emission from compressed ISM around the lobes, and (c) dynamical constraints on the immediate environment of binaries like GRS $1915+105$, which leads to the conclusion that this environment must be more tenuous than even the most under-dense regions of the ISM (and thus most likely be radio plasma too dim to detect directly).

\section{References}

Bîrzan, L., Rafferty, D. A., McNamara, B. R., Wise, M. W., \& Nulsen, P. E. J. 2004, ApJ, 607, 800

Castor, J., McCray, R., \& Weaver, R. 1975, ApJL, 200, L107

Choi, Y., Reynolds, C. S., Heinz, S., Rosenberg, J. L., Perlman, E. S., \& Yang, J. 2004, ApJ, 606, 185

Corbel, S., Fender, R. F., Tzioumis, A. K., Tomsick, J. A., Orosz, J. A., Miller, J. M., Wijnands, R., \& Kaaret, P. 2002, Science, 298, 196

\footnotetext{
${ }^{1}$ Note, however, that small opening angles below $1^{\circ}$ are not entirely implausible - see Heinz (2006)
} 
Dubner, G. M., Holdaway, M., Goss, W. M., \& Mirabel, I. F. 1998, AJ, 116, 1842

Fanaroff, B. L. \& Riley, J. M. 1974, MNRAS, 167, 31

Fender, R., Spencer, R., Tzioumis, T., Wu, K., van der Klis, M., van Paradijs, J., \& Johnston, H. 1998, ApJL, 506, L121

Fender, R. P., Garrington, S. T., McKay, D. J., Muxlow, T. W. B., Pooley, G. G., Spencer, R. E., Stirling, A. M., \& Waltman, E. B. 1999, MNRAS, 304, 865

Fender, R. P. \& Kuulkers, E. 2001, MNRAS, 324, 923

Gallo, E., Fender, R., Kaiser, C., Russell, D., Morganti, R., Oosterloo, T., \& Heinz, S. 2005, Nature, 436,819

Hardcastle, M. J. 2005, A\&A, 434, 35

Heinz, S. 2000, Ph.D. Thesis

-. 2006, ApJ, 636, 316

Heinz, S., Choi, Y., Reynolds, C. S., \& Begelman, M. C. 2002, ApJL, 569, L79

Heinz, S., Reynolds, C. S., \& Begelman, M. C. 1998, ApJ, 501, 126

Heinz, S. \& Sunyaev, R. 2002, A\&A, 390, 751

Heinz, S. \& Sunyaev, R. A. 2003, MNRAS, 343, L59

Hjellming, R. M. \& Rupen, M. P. 1995, Nature, 375, 464

Kaiser, C. R., Gunn, K. F., Brocksopp, C., \& Sokoloski, J. L. 2004, ApJ, 612, 332

Mirabel, I. F. \& Rodríguez, L. F. 1994, Nature, 371, 46

Mirabel, I. F., Rodríguez, L. F., Cordier, B., Paul, J., \& Lebrun, F. 1992, Nature, 358, 215

Reynolds, C. S., Heinz, S., \& Begelman, M. C. 2001, ApJL, 549, L179

-. 2002, MNRAS, 332, 271

Russell, D. M., Fender, R. P., Gallo, E., \& Kaiser, C. R. 2007, MNRAS, 376, 1341 